\title{
Aus- und Weiterbildung im Focus
}

\author{
Focus on Basic and Continuing Education
}

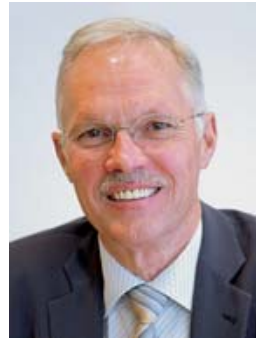

F. U. Niethard

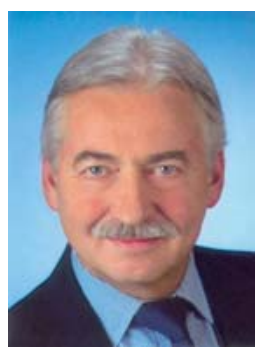

K. Weise

Bibliografie

DOI http://dx.doi.org/

10.1055/s-0030-1270824

Z Orthop Unfall 2011; 149: $15-$

16 @ Georg Thieme Verlag KG

Stuttgart · New York .

ISSN 1864-6697

Korrespondenzadressen Prof. Dr. med. Kuno Weise BG-Unfallklinik

Schnarrenbergstraße 95

72076 Tübingen

Tel.: 0 07071/606-1001

Fax: 07071/606-1002

weise@bgu-tuebingen.de

Prof. Dr. med. Fritz U. Niethard

Orthopädische Klinik

Universitätsklinik der

RWTH Aachen

Pauwelsstraße 30

52074 Aachen

Tel.: 0241/808-9410

Fax: $0241 / 808-2453$

funiethard@googlemail.com
Der Nachwuchsmangel in den chirurgischen Fächern nimmt zu. Die Kliniken klagen über einen zum Teil schon gravierenden Ärztemangel, ohne dass dessen Ursachen so ohne Weiteres benannt werden könnten. Anlässlich des 3. Zukunftsworkshops der Deutschen Gesellschaft für Orthopädie und Unfallchirurgie im November in Berlin wurde das Nebeneinander von Über- und Unterversorgung - und zur letzteren gehört der Nachwuchsmangel - diskutiert [7]. Das Paradoxon des Ärztemangels bei gleichzeitig steigenden Arztzahlen wird von der kassenärztlichen Bundesvereinigung durch die Entwicklung des medizinischen Fortschritts, den demografischen Wandel der Bevölkerung, die Feminisierung des ärztlichen Berufs $[11,13]$ und den allgemeinen Trend zur Arbeitszeitverkürzung erklärt. Im Bereich von Orthopädie und Unfallchirurgie hat sich die Zahl berufstätiger Orthopäden seit 1970 versechsfacht, seit 1990 um immerhin ca. 40\% erhöht. Die Zahl der berufstätigen Unfallchirurgen (inkl. derjenigen mit Schwerpunkt oder Teilgebiet Unfallchirurgie) hat sich seit 1990 mehr als verdoppelt. Daraus resultiert, dass Deutschland mit über 9 Orthopäden und Unfallchirurgen pro 100000 Einwohner eine der höchsten Arztdichten hinter Spanien und Italien mit sogar mehr als 12/100 000 Einwohner besitzt. Die Niederlande dagegen haben in ihrem System eine Arztdichte von ca. 4 Orthopäden und Unfallchirurgen pro 100000 Einwohner.

Woher kommt also der Nachwuchsmangel? Zahlreiche Aktivitäten der Fachgesellschaften fokussieren sich auf die Ausbildung, weil offenbar bereits dort die Entscheidung für oder gegen eine Tätigkeit in einem chirurgischen Fach fällt. Eine Studie aus der Ruhruniversität Bochum über die Zukunftspläne der Nachwuchsmediziner hatte ja gezeigt, dass unter annähernd 3800 befragten Medizinstudenten im 1 . Semester noch $34,4 \%$ in ein chirurgisches Fach und $11,2 \%$ in die Orthopädie und Unfallchirurgie gehen wollen. Mit der PJ-Zeit kommt es allerdings zu einem dramatischen Umschwung: der Wunsch, sich in ein chirurgisches Fach zu begeben, fällt von 34 auf 16\% und bei der Orthopädie von 11 auf 7\% [6]. Als wesentlicher Grund für die negative Grundeinstellung werden die Arbeitsbedingungen und $\mathrm{Ar}$ beitszeiten genannt, die in den chirurgischen Fächern von den PJlern erstmals als abschreckend wahrgenommen werden. Die Studenten antizipieren damit ein Negativbild der chirurgischen
Fächer, das offenbar nicht so sehr vom Lehrkatalog als vielmehr vom alltäglichen Arbeitsgeschehen geprägt wird.

Es ist ein besonderes Verdienst, dass sich die Arbeitsgemeinschaft „Lehre“ der Deutschen Gesellschaft für Orthopädie und Unfallchirurgie dieser Fragestellungen angenommen hat. Sie hat nicht nur einen Lernzielkatalog für das Fach Orthopädie/Unfallchirurgie vorgelegt, der 2008 fertiggestellt wurde $[1,10]$, sondern nun auch eine Befragung an den medizinischen Fakultäten und Hochschulen in Deutschland zur Ausbildung in Orthopädie und Unfallchirurgie durchgeführt [8]. Dabei zeigte sich, dass 6 Jahre nach der neuen Musterweiterbildungsordnung (im Jahr 2003), 7 Jahre nach Inkrafttreten der neuen Approbationsordnung für Ärzte (2002) und 9 Jahre nach der Deklaration der Bone and Joint Decade (2000) die studentische Ausbildung in Orthopädie und Unfallchirurgie in Deutschland nach wie vor sehr heterogen aufgebaut ist. In $63 \%$ besteht noch eine getrennte Lehre in Orthopädie und Unfallchirurgie und nur in wenigen Fakultäten wird der von der Arbeitsgemeinschaft Lehre entwickelte Lernzielkatalog genutzt. Nur an 1 Fakultät wurden Orthopädie und Unfallchirurgie als gemeinsames Fach und getrennt von der Chirurgie unterrichtet. DGOOC und DGU sind als Fachgesellschaften sehr bemüht, die „Corporate Identity“ für das neue Fach weiterzuentwickeln. Die Publikationen zu diesem Thema häufen sich [2-5,12]. Zahlreiche Aktivitäten zielen darauf hin, die Studenten für das Fach zu gewinnen: Summer School, Kongressstipendien, Promotionsstipendien, Tutorenprogramme vor Ort sind eingerichtet worden, um die Studenten für Orthopädie und Unfallchirurgie zu begeistern. Aber bleibt dies alles nicht Makulatur, wenn die Gemeinsamkeit des Faches in der Lehre und in der praktischen Ausbildung, vor allem in der PJ-Zeit, nicht gelebt wird?

Die Gründe für ein Beibehalten der vormaligen Regelung sind vielfach diskutiert: die Orthopädie vermag als Wahlfach eigene PJ-Studenten anzuziehen, die Unfallchirurgie erhält als Bereich des Gebiets Chirurgie anteilig PJ-Studenten aus der chirurgischen Lehre. Damit wird Orthopädie und Unfallchirurgie von beiden Seiten bestückt und scheint zahlenmäßig einen Gewinn aufzuweisen. $\mathrm{Zu}$ hinterfragen ist allerdings, ob dies nicht doch zu einem Imageverlust des Faches führt, der sich bei der endgültigen Berufswahl negativ auswirkt. 
Ein Ärztemangel in den chirurgischen Fächern und darunter auch in Orthopädie und Unfallchirurgie hat nichts mit den Studentenzahlen zu tun; denn diese reichen aus, um die ärztliche Versorgung auf gleich hohem Niveau auch in Zukunft zu gewährleisten [9].

Offensichtlich ist dies vielmehr ein Verteilungsproblem, das ganz wesentlich während der PJ-Zeit entschieden wird. Bedauerlicherweise sind genaue Zahlen speziell für Orthopädie und Unfallchirurgie nicht vorhanden.

Die Aufmerksamkeit der Fachgesellschaften muss sich aber auf diesen Aspekt richten, denn die demografische Entwicklung geht auch an der Ärzteschaft in Orthopädie und Unfallchirurgie nicht spurlos vorbei. In 15 Jahren wird ein großer Teil der noch aktiv tätigen Ärzte ausgeschieden sein. Der Bedarf für Ärzte in $\mathrm{O}+\mathrm{U}$ wird nicht geringer: Aus- und Weiterbildung stehen weiter im Focus.

\section{F.U. Niethard, Aachen}

K. Weise, Tübingen

\section{Literatur}

1 Dreinhöfer KE, Walcher F, Obertacke U et al. Entwicklung des Lernzielkatalogs „Muskuloskeletale Erkrankungen, Verletzungen und traumatische Notfälle“ für Orthopädie-Unfallchirurgie im Medizinstudium. Z Orthop Unfall 2008; 146: 520-533
2 Niethard FU. Wie wichtig ist die Orthopädie für die Studenten? Z Orthop 1999; 137: 199-200

3 Niethard FU, Puhl W. Die „Bone and Joint Decade 2000-2010“. Z Orthop 1999; 137: 1

4 Niethard FU. Weiterbildung und Arbeitszeitgesetz: Wie werden aus Ärzten gute Orthopäden? Z Orthop 2001; 139: 467-468

5 Niethard FU, Siebert $H$. Nachwuchsmangel wegen gefühlter Unzufriedenheit? Orthopädie Mitteilungen 2009; 3: 330-331

6 Niethard FU, Weise K. Weiterbildung auf dem Prüfstand. Z Orthop Unfall 2010; 148: 385-386

7 Niethard FU, Siebert H. Über- und Unterversorgung in Orthopädie und Unfallchirurgie. Orthopädie Mitteilungen 2011, 1: 61-63

8 Rüsseler MU, Obertacke K, Dreinhöfer C et al. Die studentische Lehre im gemeinsamen Fach Orthopädie-Unfallchirurgie - eine deutschlandweite Statuserhebung. Z Orthop Unfall 2011; 149: 27-32

9 Schäfer $R$. Wieviele Ärzte braucht das Land? Vortrag beim Zukunftsworkshop der DGOU in Berlin; 30.11.2011

10 Walcher F, Dreinhöfer KE, Obertacke U et al. Entwicklung des Lernzielkatalogs „Muskuloskeletale Erkrankungen, Verletzungen und traumatische Notfälle“ für Orthopädie-Unfallchirurgie im Medizinstudium. Unfallchirurg 2008; 111: 670-687

11 Weise K, Niethard FU. Die so genannte Feminisierung der Medizin - unter spezieller Fokussierung auf die Nachwuchsgewinnung in Unfallchirurgie und Orthopädie. Z Orthop Unfall 2010: 148: 273-275

12 Weise K, Niethard FU. Das junge Forum der DGOU - über die Wünsche und Vorschläge des Nachwuchses in Unfallchirurgie und Orthopädie. Z Orthop Unfall 2009; 147: 15-16

13 Weise K, Niethard FU. Zum Nachwuchsmangel in Unfallchirurgie und Orthopädie. Z Orthop Unfall 2010; 148: 17-18 